\title{
Entomopathogenic potential of Purpureocillium lilacinum against the model insect Galleria mellonella (Lepidoptera: Pyralidae)
}

\author{
Sümeyra Nur Şanal Demirci ${ }^{1 *}$, Hülya Altuntaş ${ }^{2}$ \\ ${ }^{1}$ Eskisehir Technical University, Graduate School of Sciences, Department of Biology,Turkey \\ ${ }^{2}$ Eskisehir Technical University, Faculty of Sciences, Department of Biology,Turkey \\ ${ }^{\star}$ Corresponding author, E-mail: sumeyrasanal@gmail.com
}

\begin{abstract}
Pathogenicity of the entomopathogenic fungus Purpureocillium lilacinum against Galleria mellonella larvae was studied using two application methods, immersion and injection. G. mellonella last instar larvae were immersed and injected with fungal suspensions of different conidial concentrations $\left(1 \times 10^{3}, 1 \times 10^{4}, 1 \times 10^{5}, 1 \times 10^{6}, 1 \times 10^{7}\right.$ and $1 \times 10^{8}$ conidia $\left.\mathrm{mL}^{-1}\right)$ and mortality was recorded daily. P. lilacinum showed pathogenicity at the different concentrations by both methods. P. lilacinum was highly infectious for G. mellonella causing $100 \%$ larval mortality within 7 days post-immersion with $1 \times 10^{8}$ concentrations. The median lethal time $\left(\mathrm{LT}_{50}\right)$ was 1.83 days. Using the injection method, $1 \times 10^{8}, 1 \times 10^{7}, 1 \times 10^{6}$ and $1 \times 10^{5}$ concentrations caused $100 \%$ larval mortality within $1,2,3$ and 4 days after injection respectively. The median lethal time $\left(\mathrm{LT}_{50}\right)$ was $1,1.47,1.96$, aand 2.05 days, respectively. For both methods (immersion and injection) the lethal concentration of P. lilacinum that caused $50 \%$ larval mortality $\left(\mathrm{LC}_{50}\right)$ was $3.1 \times 10^{4}$ and $4.7 \times 10^{3} \mathrm{conidia}^{\mathrm{mL}} \mathrm{m}^{-1}$, respectively. Both methods were effective on larvae, but the injection method was more effective than immersion method at $4.7 \times 10^{3}$ conidia $\mathrm{mL}^{-1}$ causing $50 \%$ larval mortality. For immersion and injection methods, the median lethal times $\left(\mathrm{LT}_{50}\right)$ at the concentrations of $1.0 \times 10^{8}$ conidia $\mathrm{mL}^{-1}$ were 1.83 and 1 days, respectively. The pathogenicity results obtained from this isolate using the model insect G. mellonella showed that $P$. lilacinum can be used as a potential biopesticidal agent against lepidopteran pests.
\end{abstract}

Key words: biopesticidal activity, entomopathogen fungus, Lepidopteran pest, pathogenicity. Abbreviations: $\mathrm{LT}_{50}, 50 \%$ lethal time; $\mathrm{LC}_{50}, 50 \%$ larval mortality.

\section{Introduction}

It is known that insects, one of the most important elements of biological diversity, have various biological and nonbiological factors affecting their growth and development in the environment. One of the important factors that insects are exposed to during the development process is entomopathogenic fungi. Entomopathogenic fungi are also known to be more effective in the control of various insect pests, compared to other microorganisms (Deacon 1983). Previous studies reported that several species of entomopathogenic fungi such as Beauveria bassiana, Isaria fumosorosea, Lecanicillium lecanii, Metarhizium anisopliae, Paecilomyces lilacinus and Pandora neoaphidis, have shown good potential for biological control of insects and root-knot nematode (Hajek, Leger 1994; Klingen et al. 2002; Kiewnick, Sikora 2006; Ibrahim et al. 2016; Majeed et al. 2017). These abovementioned studies suggested that entomopathogenic fungus could be used in integrated pest management programmes as eco-friendly biopesticides. For this reason, in this study, we selected Purpureocillium lilacinum (Thom) Luangsa-ard, Houbraken, Hywel-Jones, and Samson (KUKENS WDCM101) [the old name is
Paecilomyces lilacinus (Thom)], an entomopathogenic fungus and a common soil fungus (Demirel et al. 2005). P. lilacinum has been used as a biocontrol agent against eggs of plant root nematodes (Khan et al. 2003). Several studies about the entomopathogenic effects of Paecilomyces species showed that P. lilacinus, Paecilomyces farinosus and Paecilomyces fumosoroseus have pathogenic potential on some insect pests (Meitkiewski et al. 1997; Pedro et al. 2001; James 2003; Michalaki et al. 2007). Also, it has been shown that different strains of $P$. lilacinus have entomopathogenic effects against some insects (Esser and El-Gholl 1993; Bustillo et al. 1999; Beron and Diaz 2005; Ghazavi et al. 2005). Therefore, the first aim of this study was to test the entomopathogenic effects of P. lilacinum, isolated from agricultural lands of Eskişehir province, on the model insect Galleria mellonella (Lepidoptera: Pyralidae) last instar larvae.

To investigate the pathogenicity of $P$. lilacinum on the insects, we selected the greater wax moth, G. mellonella larvae because of two reasons. First, this species causes significant economic losses in the beekeeping industry by feeding on honeycomb by larval stages and laying eggs in the adult stage on the honeycombs of Apis mellifera 
(Hymenoptera: Apidae) during storage (Charriere, Imdorf 1997). Several fumigant insecticides such as sulphur dioxide, acetic acid, formic acid, para-dichloro benzene, methyl bromide and phosphine, have been used to control wax moth on beeswax combs during storage. Use of these chemicals is harmful to bee populations (Kwadha et al. 2017). Therefore, we think that eco-friendly pesticides, such as entomopathogenic fungus, could be used as an alternative bioinsecticide. Second, G. mellonella is an excellent model insect used in pathogenic and toxicological investigations of various xenobiotics, and also quite easy to grow in the laboratory (Altınçiçek et al. 2007; Altuntaş et al. 2016; Maguire et al. 2016). Furthermore, the second aim of this study was to determine the pathogenicity of P. lilacinum against G. mellonella larvae using immersion and injection methods. Thus, we also determined which application method was more effective.

\section{Materials and methods}

\section{Fungus culture}

The entomopathogenic fungus Paecilomyces lilacinum was isolated from the agricultural lands of Eskişehir province, Turkey and was identified according to Pitt (1978) by Rasime Demirel (Demirel et al. 2005). This strain is also maintained in the KUKENS culture collection (WDCM101). The isolated strain of P. lilacinum was grown on malt extract agar at $28^{\circ} \mathrm{C}$ in the dark. Conidial suspensions for assays were prepared by scraping conidia from 14-day-old well sporulated cultures into an aqueous solution of $1 \%$ Tween 80 . Different conidial concentrations $\left(1 \times 10^{3}, 1 \times 10^{4}, 1 \times 10^{5}, 1 \times 10^{6}, 1 \times 10^{7}\right.$ and $1 \times 10^{8}$ conidia $\mathrm{mL}^{-1}$ ) were determined using a Neubauer hemocytometer.

\section{Insect rearing}

A Galleria mellonella colony was maintained by feeding the insects on an artificial diet including $340 \mathrm{~g}$ bran, $20 \mathrm{~g}$ pollen, $75 \mathrm{~mL}$ filtered honey, $150 \mathrm{~mL}$ glycerol, 100 g ground old dark honeycomb and $75 \mathrm{~mL}$ distilled water, modified by Bronskill (1961). All stock and experimental laboratory cultures were maintained at Anadolu University, Eskişehir,
Turkey at $25 \pm 2{ }^{\circ} \mathrm{C}, 60 \pm 5 \%$ relative humidity, and constant darkness.

\section{Bioassay of P. ilacinum}

Pathogenicity of the entomopathogenic fungus Purpureocillium against the G. mellonella larvae was studied based on immersion and injection methods. Infection of $G$. mellonella last instar larvae was carried out by immersion at selected conidial concentrations $\left(1 \times 10^{3}, 1 \times 10^{4}, 1 \times\right.$ $10^{5}, 1 \times 10^{6}, 1 \times 10^{7}$ and $1 \times 10^{8}$ conidia $\mathrm{mL}^{-1}$ ) for $5 \mathrm{~s}$. Also, the last instar larvae were immersed in an only aqueous solution of $1 \%$ Tween 80 as a control $(n=100)$. Using the injection method, last instar larvae of G. mellonella were treated by injection with $10 \mu \mathrm{L}$ of each conidial suspension of different conidial concentrations $\left(1 \times 10^{3}, 1 \times 10^{4}, 1 \times 10^{5}\right.$, $1 \times 10^{6}, 1 \times 10^{7}$ and $1 \times 10^{8}$ conidia $\mathrm{mL}^{-1}$ ) and also $10 \mu \mathrm{L}$ of the aqueous solution of $1 \%$ Tween 80 was used as a control $(n=100)$. Infected larvae were incubated at $25{ }^{\circ} \mathrm{C}$ in the dark for 15 days. Using both methods, mortality rates were observed daily for 15 days and recorded. All assays were replicated five times with 20 larvae completely randomized from different populations.

\section{Statistical analysis}

The median lethal time (LT50) was calculated according to Beron and Diaz (2005) and 100\% mortality was determined in both methods. All observed mortality percentages were corrected using Abbott's formula (1925). According to the larval mortality data, the median lethal concentrations of $P$. lilacinum with associated 95\% confidence levels $(P<0.05)$ for both methods were determined using Probit Analysis in Statistical Package for the Social Sciences (SPSS) software (version 18.0 for Windows, SPSS Science, Chicago, IL).

\section{Results and discussion}

In this study, it was determined that the entomopathogenic activity of $P$. lilacinum against G. mellonella larvae differed according to the application method (Table 1 and 2). $P$. lilacinum caused high mortality for G. mellonella resulting in approximately $100 \%$ mortality at 7 days post-immersion

Table 1. Percentage of mortality and median lethal time $\left(\mathrm{LT}_{50}\right)$ values of P. lilacinum suspensions against last instar larvae of G. mellonella. ${ }^{\star}$ Mortality percentages (\%) were corrected using Abbott's formula (1925). ${ }^{\star *}$ The Median lethal Time $\left(\mathrm{LT}_{50}\right)$ was calculated by $\Sigma\left(\right.$ days $_{\mathrm{n}} \times$ infected larvae ${ }_{\mathrm{n}}$ ) / total of infected larvae (Beron, Diaz 2005)

\begin{tabular}{|c|c|c|c|}
\hline Application method & $\begin{array}{l}\text { Fungal concentration } \\
\quad\left(\text { conidia } \mathrm{mL}^{-1}\right)\end{array}$ & Mortality $(\%)^{*}$ & $\mathrm{LT}_{50}(\text { days })^{\star *}$ \\
\hline \multirow[t]{3}{*}{ Immersion } & $1 \times 10^{6}$ & 60 & 2.66 \\
\hline & $1 \times 10^{7}$ & 74 & 2.61 \\
\hline & $1 \times 10^{8}$ & 100 & 1.83 \\
\hline \multirow[t]{5}{*}{ Injection } & $1 \times 10^{4}$ & 52 & 2.88 \\
\hline & $1 \times 10^{5}$ & 100 & 2.05 \\
\hline & $1 \times 10^{6}$ & 100 & 1.96 \\
\hline & $1 \times 10^{7}$ & 100 & 1.47 \\
\hline & $1 \times 10^{8}$ & 100 & 1.00 \\
\hline
\end{tabular}


Table 2. Mortality percentage of G. mellonella larvae exposed to different condial suspansions of P. lilacinum in both application methods

\begin{tabular}{|c|c|c|c|c|c|}
\hline \multirow{2}{*}{$\begin{array}{l}\text { Fungal } \\
\text { concentration } \\
\text { (conidia } \mathrm{mL}^{-1} \text { ) }\end{array}$} & \multirow[t]{2}{*}{ Log concentration } & \multicolumn{2}{|c|}{ Mortality (\%) } & \multicolumn{2}{|c|}{ Corrected mortality (\%) } \\
\hline & & Immersion & Injection & Immersion & Injection \\
\hline Control & 0 & 1 & 1 & 1 & 1.0 \\
\hline $1 \times 10^{3}$ & 3 & 40 & 27 & 40 & 27.0 \\
\hline $1 \times 10^{4}$ & 4 & 45 & 52 & 45 & 52.0 \\
\hline $1 \times 10^{5}$ & 5 & 48 & 100 & 48 & 97.5 \\
\hline $1 \times 10^{6}$ & 6 & 60 & 100 & 60 & 97.5 \\
\hline $1 \times 10^{7}$ & 7 & 74 & 100 & 74 & 97.5 \\
\hline $1 \times 10^{8}$ & 8 & 100 & 100 & 98 & 97.5 \\
\hline
\end{tabular}

with $1 \times 10^{8}$ conidial concentration. However, fungal concentrations at $1 \times 10^{8}, 1 \times 10^{7}, 1 \times 10^{6}$ and $1 \times 10^{5}$ conidia $\mathrm{mL}^{-1}$ caused approximately $100 \%$ mortality after injection at 1,2,3 and 4 days respectively (Table 1 ). At the same time, we observed $100 \%$ larval mortality at $1 \times 10^{8}$ conidia $\mathrm{mL}^{-1}$ in immersion and injection methods and the median lethal time $\left(\mathrm{LT}_{50}\right)$ was determined at 1.83 and 1 days, respectively. The $\mathrm{LT}_{50}$ values of fungal concentrations at $1 \times 10^{7}, 1 \times 10^{6}$ and $1 \times 10^{5}$ conidia $\mathrm{mL}^{-1}$ were $1.47,1.96$ and 2.05 days, respectively using the injection method for G. mellonella larvae (Table 1). For the immersion method, the $\mathrm{LT}_{50}$ values of fungal concentrations at $1 \times 10^{7}$ and 1 $\times 10^{6}$ conidia $\mathrm{mL}^{-1}$ fungal suspensions were 2.61 and 2.66 days, respectively (Table 1 ). Similar to the results of our study, Ibrahim et al. (2016) reported that P. lilacinum at the high concentration of $10^{9}$ conidia $\mathrm{mL}^{-1}$ applied with immersion method caused $87.5 \%$ mortality of G. mellonella larvae and also $\mathrm{LT}_{50}$ was 2.2 days. However, in a previous study using the agar surface technique, P. lilacinus had very low infection with $30 \%$ larval mortality on G. mellonella in 10 days and a $\mathrm{LT}_{50}$ value 16.16 days (Baydar et al. 2016). the reason for the differences between this previous study and our study could be due to application method and fungus strain. Based on these results, we conclude that our strain of P. lilacinum had the shortest $\mathrm{LT}_{50}$ values against last instar larva of $G$. mellonella using the applied two methods.

The percentage of larval mortality using the tested methods at different conidial concentrations of P. lilacinum is summarized in Table 2. Our findings showed that fungal concentration of $1 \times 10^{8}$ conidia $\mathrm{mL}^{-1}$ caused $100 \%$ larval mortality using immersion method. On using the injection method, $100 \%$ larval mortality occured at $1 \times 10^{5}$ conidia $\mathrm{mL}^{-1}$. Probit analysis showed that the $\mathrm{LC}_{50}$ value of the conidial concentration of P. lilacinum was $3.1 \times$ $10^{4}$ (confidence limit: $2.3 \times 10^{4}-5.4 \times 10^{5}$ ) condia $\mathrm{mL}^{-1}$ $\left(\chi^{2}=27.041 ; d f=4 ; P=0.000\right)$ for the immersion method. For the the incetion method, $\mathrm{LC}_{50}$ value of the conidial concentration of $P$. lilacinum was $4.7 \times 10^{3}$ (confidence limit: $\left.1.4 \times 10^{3}-1.3 \times 10^{4}\right)$ conidia $\mathrm{mL}^{-1}\left(\chi^{2}=16.89 ; d f=4\right.$; $P=0.002)$. The present findings indicate that the effective virulence of $P$. lilacinum against $G$. mellonella larvae differed between the application methods. In other words, the injection of fungal suspension was more effective than the immersion bioassay for model insect G. mellonella.

A previous study reported that bioassay isolate PDRL812 of P. lilacinus showed higher mortality rates on mustard aphid (Lipaphis erysimi) with a $\mathrm{LC}_{50}$ value at $5.1 \times$ $10^{3}$ spores $\mathrm{mL}^{-1}$ and $100 \%$ mortality of insect population at $10^{7}$ spores $\mathrm{mL}^{-1}$ (Ujjan, Shahzad 2012). In agreement with the above-mentioned study, we observed almost similar $\mathrm{LC}_{50}$ values using the injection method in G. mellonella. Our findings are also in line with the findings of Ibrahim et al. (2016) who reported that different fungal isolates including $P$. lilacinus caused varying mortality rates of $2^{\text {nd }}$ instar larvae of $G$. mellonella at different times with immersion methods. On the other hand, it was noted that $P$. fumosoroseus was more virulent than P. lilacinus on white fly nymphs Trialeurodes vaporariorum (Gokce, Er 2005). Mwamburi et al. (2010) also found that P. lilacinus was non-pathogenic to adult house flies Musca domestica. Pathogenicity differences of P. lilacinum may be associated with the application method and the different conidia concentrations attached to the cuticle of the studied insect groups (Clarkson, Charnley 1996; Rambadan et al. 2011). Since we eliminated the cuticle barrier with the injection method, $P$. lilacinum was more pathogenic against $G$. mellonella at lower concentrations and short exposure times. Furthermore, the present results obtained with the model insect G. mellonella indicate that P. lilacinum may be used for biological control of the lepidopteran pests instead of dipterous pests.

In conclusion, the obtained results showed that this isolate of $P$. lilacinum had high pathogenicity on $G$. mellonella larvae considering the low $\mathrm{LT}_{50}$ and $\mathrm{LC}_{50}$ values. These fungal formulations would be beneficial for field studies in future. Therefore, we suggest that further research is done to determine the efficacy and viability of this isolate for longer periods in field or storage environments as a contact biopesticide against target lepidopterous pests. Considering human and environmental health, biopesticides obtained from entomopathogenic fungi could be used as alternative biocontrol agents in integrated pest management programmes. Thus, research on the use of entomopathogenic fungi for the control of insect pests should be continued. 


\section{Acknowledgements}

The Purpureocillium lilacinum (KUKENS WDCM101), used for the present study, was kindly provided by Dr. Rasime Demirel. The authors are also very grateful to R. Demirel for her technical support.

\section{References}

Abbott W.S. 1925. A method of computing the effectiveness of an insecticide. J. Econ Entomol. 18: 265-267.

Altınçiçek B., Lindner M., Linder D., Preissner K., Vilcinskas A. 2007. Microbial metalloproteinases mediate sensing of invading pathogens and activate innate immune responses in the lepidopteran model host Galleria mellonella. Insect Immun. 75: 175-183.

Altuntaş H., Duman E., Şanal Demirci S.N., Ergin E. 2016. Toxicological and physiological effects of ethephon on the model organism, Galleria mellonella L. 1758 (Lepidoptera: Pyralidae). Türk. Entomol. Derg. 40: 413-423.

Baydar R., Güven Ö., Karaca I. 2016. Occurence of Entomopathogenic fungi in agricultural soils from Isparta province in Turkey and their pathogenicity to Galleria mellonella (L.) (Lepidoptera: Pyralidae) larvae. Egypt. J. Biol. Pest Contr. 26: 323-327.

Beron C.M., Diaz B.M. 2005. Pathogenicity of hyphomycetous fungi against Cyclocephala signaticollis. Biocontrol 50: 143150.

Bronskill J.F. 1961. A cage to simplify the rearing of the greater wax moth, Galleria mellonella (Pyralidae). J. Lepidopt. Soc. 15: 102-104.

Bustillo A., Martha Bernal G., Benavides P., Chaves B. 1999. Dynamics of Beauveria bassiana and Metarhizium anisopliae infecting Hypothenemus hampei (Coleoptera: Scolytidae) populations emerging from fallen coffee berries. Florida Entomol. 82: 491-498.

Charriere J.D., Imdorf A. 1997. Protection of Honey Combs from Moth Damage. Liebefeld, Bern, Switzerland.

Clarkson J.M., Charnley A.K. 1996. New insight into the mechanisms of fungal pathogenesis in insects. Trends Microbiol. 4: 197-203.

Deacon J.W. 1983. Microbial Control of Pests and Diseases. American Society for Microbiology, New York.

Demirel R., Ilhan S., Asan A., Kınacı E., Oner S. 2005. Microfungi in cultivated fields in Eskişehir provience (Turkey). J. Basic Microbiol. 45: 279-293.

Esser R.P., El-Gholl N.E. 1993. Paecilomyces lilacinus, a fungus that parasitizes nematode eggs. Nematology Circular No. 203.

Ghazavi M., Zangeneh S., Abaii M. 2005. New records of some entomopathogenic fungi. Iran Rostaniha 6: 43-46.

Gokce A., Er M.K. 2005. Pathogenicity of Paecilomyces spp., to the glass house whitefly, Trialeurodes vaporariorum, with some observations to the fungal infection process. Turk. J. Argicult. 29: 331-339.

Hajek A.E., Leger R.J.S.T. 1994. Interactions between fungal pathogens and insects hosts. Annu. Rev. Entomol. 39: 293-322.

Maguire R., Duggan O., Kavanagh K. 2016. Evaluation of Galleria mellonella larvae as an in vivo model for assessing the relative toxicity of food preservative agents. Cell Biol. Toxicol. 32:
209-216.

Majeed M.Z., Fiaz M.,Ma C.S.,Afzal M.2017.Entomopathogenicity of three Muscardine Fungi, Beauveria bassiana, Isaria fumosorosea and Metarhizium anisopliae, against the Asian Citrus Psyllid, Diaphorina citri Kuwayama (Hemiptera: Psyllidae). Egypt. J. Biol. Pest Contr. 27: 211-215.

Meitkiewski R.T., Pell J.K., Clark S.J. 1997. Influence of pesticide use on the natural occurrence of entomopathogenic fungi in arable soils in the UK: Field and laboratory comparisons. Biocontrol Sci. Technol. 565-757.

Ibrahim A.A., Mohamed H.F., El-Naggar S.E.M., Swelim M.A., Elkhawaga O.E. 2016. Isolation and selection of entomopathogenic fungi as biocontrol agent against the greater wax moth, Galleria mellonella L. (Lepidoptera: Pyralidae). Egypt. J. Biol. Pest Contr. 26: 249-253.

James R.R. 2003 Combining azadirachtin and Paecilomyces fumosoroseus (Deuteromycotina: Hyphomycetes) to control Bemisia argentifolii (Homoptera: Aleyrodidae). J. Econ. Entomol. 96: 25-30.

Klingen I., Meadow R., Aandal T. 2002. Mortality of Delia floralis, Galleria mellonella and Mamestra brassicae treated with insect pathogenic hyphomycetous fungi. J. Appl. Entomol. 126: 231237.

Khan A., Williams K., Nevalainen H. 2003. Testing the nematophagous biological control strain Paecilomyces lilacinus 251 for paecilotoxin production. FEMS Microbiol. Lett. 227: 107-111.

Kiewnick S., Sikora R.A. 2006. Biological control of the root-knot nematode Meloidogyne incognita by Paecilomyces lilacinus strain 251. Biol. Contr. 38:179-187.

Kwadha C.A., Ong'amo G.O., Ndegwa P.N., Raina S.K., Fombong A.T. 2017. The biology and control of the greater wax moth, Galleria mellonella. Insects 8: 1-17.

Michalaki M.P., Athanassiou G., Steenberg T., Buchelos C.Th. 2007. Effect of Paecilomyces fumosoroseus (Wise) Brown and Smith (Ascomycota: Hypocreales) alone or in combination with diatomaceous earth against Tribolium confusum Jacquelin du Val (Coleoptera: Tenebrionidae) and Ephestia kuehniella Zeller (Lepidoptera: Pyralidae). Biol. Contr. 40: 280-286.

Miller L.C., Tainter M.L. 1944. Estimation of $\mathrm{LD}_{50}$ and its error by means of log-probit graph paper. Proc. Soc. Exp. Biol. Med. 57: 261.

Mwamburi L.A., Laing M.D., Miller R.M. 2010. Laboratory screening of insecticidal activities of Beauveria bassiana and Paecilomyces lilacinus against larval and adult house fly (Musca domestica L.). Afr. Entomol. 18: 38-46.

Pedro M.O.J., Neves E.H., Paulo T.T., Alcides M.J.R. 2001. Compatibility of entomopathogenic fungi with neonicotinoid insecticides. Neotrop. Entomol. 30: 263-268

Pitt J.I. 1979. The Genus Penicillium and its Telemorphic states Eupenicillium and Talaromyces. Academic Pres, London.

Rambadan S., Jugmohan H., Khan A. 2011. Pathogenicity and haemolymph protein changes in Edessa meditabunda $\mathrm{F}$. (Hemiptera:Pentatomidae) infected by Paecilomyces lilacinus. J. Biopest. 4: 169-175

Ujjan A.A., Shahzad S. 2012. Use of entomopathogenic fungi for the control of mustard aphid (Lipaphis erysimi) on canola (Brassica napus L.). Pak. J. Bot. 44: 2081-2086. 